\title{
Pensiero progettuale: Innovazione e protezione delle idee
}

\section{Design Thinking: Innovation and protection of ideas}

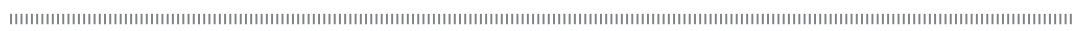

Amparo de las Mercedes Álvarez Meythaler

Facultad de Arquitectura, Diseño y Artes (FADA).

Pontificia Universidad Católica del Ecuador

gide@puce.edu.ec

\section{Rubén Méndez Reátegui}

Facultad de Jurisprudencia. Pontificia Universidad Católica del Ecuador rcmendez@puce.edu.ec

\section{Riassunto}

A partire da un approccio di Design Thinking, questo articolo mira a spiegare come i processi creativi nelle organizzazioni, espressi in un contesto di effervescenza dei diritti di proprietà, richiedono la formulazione di metodi più rigorosi per ottenere e valutare le informazioni, che permettono l'attenuazione degli effetti asimmetrici e la perdita di efficienza ed efficacia degli sforzi fatti dalle aziende. Si analizzano tre possibili interventi affinché il processo creativo generi un prezioso know-how. Le idee possono ricevere un trattamento organizzato durante le fasi iniziali: guida del pensiero creativo, uso della tecnologia attraverso strumenti di registro digitale, e strategie aziendali sostenibili focalizzate sul comportamento umano. Si conclude che partire da un pensiero progettuale richiede la sistematizzazione e l'incorporazione di un maggior rigore nel processo creativo per facilitare i processi di registrazione dei diritti di proprietà su un prodotto originale dell'organizzazione.

Parole chiave: Design Thinking, diritti di proprietà, tecnologia digitale, metodologia.
Artículo original / Original Article

Correspondencia / Correspondence gide@puce.edu.ec

rcmendez@puce.edu.ec

Financiación / Fundings Financiación: Proyecto internacional “085- UIO- 2020 Mejora Regulatoria y RIA (Análisis de Impacto Regulatorio) en Ecuador, Chile y Perú, Fase II" del GIDE-PUCE y la Carrera de Diseño de Productos(FADA) en colaboración con la U. Autónoma de Chile (Programa de Doctorado en Derecho - Línea de Investigación en Reforma del Estado).

Recibido / Received: 30/05/2021

Aceptado / Accepted: 17/09/2021

Publicado / Published: 17/12/2021

Cómo citar este trabajo How to cite this paper

Álvarez Meythaler, A., Méndez Reátegui, R. (2021). Design Thinking: Innovation and protection of ideas. i+Diseño. Revista Internacional de Innovación, Investigación y Desarrollo en Diseño, 16. DOI: 10.24310/ Idiseno.2021.v16i.13084 


\section{Resumen}

Desde un enfoque de Design Thinking, este artículo pretende explicar cómo los procesos creativos en las organizaciones, expresados en un contexto de efervescencia de los derechos de propiedad, requieren la formulación de métodos más rigurosos de obtención y evaluación de la información, que permitan mitigar los efectos asimétricos y la pérdida de eficiencia y eficacia de los esfuerzos realizados por las empresas. Se analizan tres posibles intervenciones para que el proceso creativo genere conocimientos valiosos. Las ideas articuladas pueden recibir un tratamiento organizado desde fases iniciales (lo cual puede involucrar la orientación del pensamiento creativo, el uso de la tecnología a través de herramientas de registro digital y de estrategias comerciales sostenibles centradas en el comportamiento humano). Se concluye que partir de un pensamiento de diseño requiere sistematizar e incorporar mayor rigor en el proceso creativo para facilitar los procesos de registro de derechos de propiedad sobre un producto original de la organización.

Palabras clave: Design Thinking, derechos de propiedad, tecnología digital, metodología.

\section{Abstract}

From a Design Thinking approach, this article aims to explain how creative processes in organizations, expressed within a context of the effervescence of property rights, require the formulation of more rigorous methods of obtaining and evaluating the information, which allow the mitigation of asymmetric effects and loss of efficiency and effectiveness of the efforts made by companies. Three possible interventions are analyzed so that the creative process generates valuable know-how. Ideas can receive organized treatment during the initial phases: guidance of creative thinking, use of technology through digital registry tools, and sustainable business strategies focused on human behavior. It is concluded that starting from a design thinking requires systematizing and incorporating greater rigor in the creative process to facilitate the registration processes of property rights over an original product of the organization.

Keywords: Design Thinking, property rights, digital technology, methodology.

\section{Introduzione}

Questo articolo spiega come i processi creativi nelle organizzazioni, espressi in un contesto di effervescenza dei "diritti di proprietà» (intesi in un ampio senso giuridico-economico), richiedano metodi più rigorosi per ottenere e valutare informazioni che attenuino gli effetti asimmetrici e la perdita di efficienza ed efficacia. Le prime fasi del Design Thinking coinvolgono vari tipi di informazioni soggettive che dimostrano la partecipazione e l'originalità delle idee sfidanti nello sviluppo di un nuovo prodotto. L' articolo analizza tre possibili procedure che permetterebbero al processo creativo di generare un know-how utile, e da questo le idee possono essere brevettate durante le fasi iniziali. Queste fasi creano contenuti interessanti per l'azienda e allo stesso modo rappresentano fattori di differenziazione per ogni organizzazione. La prima procedura, il cui obiettivo è il pensiero creativo basato su obiettivi aziendali a priori, genera indicatori per misurare i risultati organizzativi. Nel secondo, gli strumenti di registrazione digitale sono utilizzati per creare idee oggettive (una sorta di sistema di gestione dei «diritti di proprietà» non concreto). Infine, dal punto di vista del management, dobbiamo elaborare strategie aziendali sostenibili, progettate tenendo conto del comportamento umano, per creare incentivi collettivi che promuovano l'esercizio 
creativo tra i membri dell'azienda; cioè, con l'assegnazione efficiente ed efficace di risorse scarse quando i diritti di proprietà materiali e immateriali si moltiplicano.

\section{Proiezioni di pensiero progettuale}

A partire dal XIX secolo, la proiezione della pratica professionale del Bauhaus Design ha subito varie tappe disciplinari e transdisciplinari in tutto il mondo.

Questo slancio ha portato alla partecipazione dei designer in attività e servizi organizzativi -dalla pianificazione alla logistica- contribuendo così agli obiettivi specifici dell'azienda. Nel XXI secolo, il design si è affermato come parte della filosofia delle grandi aziende.

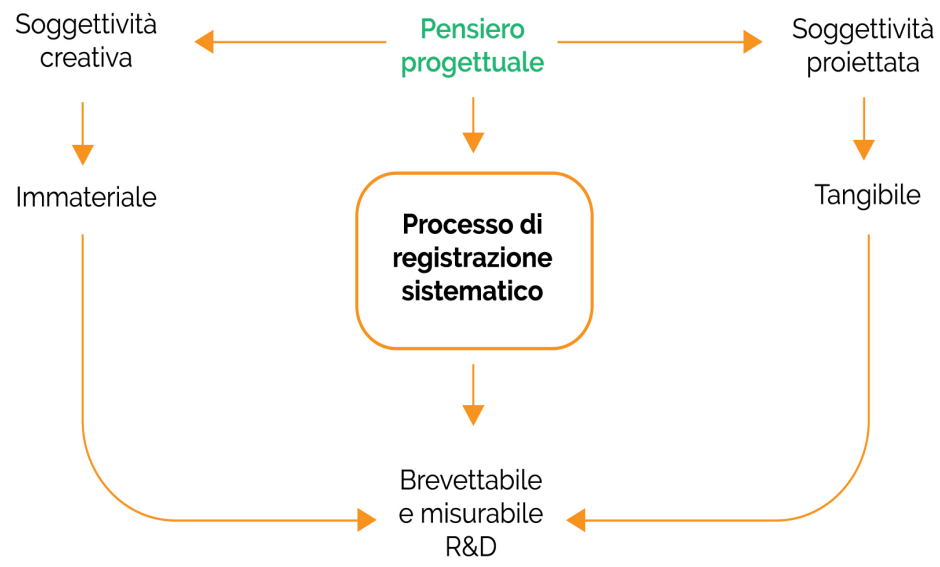

Questa trasformazione ha generato contributi da parte dei professionisti del design nelle decisioni logiche e strategiche di pianificazione relative alla maggiore esplorazione mentale di come un migliore "pensiero progettuale» ${ }^{1}$ contribuisca a creare esperienze più intelligenti, più piacevoli e più godibili che siano più significative per tutti gli attori.

Il Design Thinking è una forma di risolvere un problema complesso come farebbe un designer (Dorst, 2019). È anche emerso come lo strumento più popolare focalizzato sulla promozione più efficace dell'innovazione nelle organizzazioni. È nato insieme al movimento Open Innovation, che, sebbene sia stato attivamente indagato negli anni '80 e '90, ha ottenuto una trazione più eccellente quando I'Università di Stanford ha iniziato a insegnare «Engineering Design Thinking» nel 2005.

Il design sta emergendo come una disciplina nascente di ragionamento e argomentazione pratica con le nuove dimensioni scoperte, guidata da un designer capace di esplorare la molteplicità dei temi, tra cui la comunicazione, la costruzione, la pianificazione strategica e l'interazione sistemica. Il design sta quindi cambiando la cultura attraverso manifestazioni esterne di una natura interna o di un'organizzazione (Buchanan, 1992, p.5).

1. Secondo Tim Brown (2015), il design thinking si concentra sulle persone e sull'innovazione basata sulla cassetta degli attrezzi del designer per integrare i bisogni delle persone, le possibilità della tecnologia e i requisiti per il successo del business (IDEO) (citato da Turnali, 2015).
Figura 1. Il contributo della creatività e del design alle aziende Fonte: Elaborazione propria 
Molte organizzacioni hanno adottato il Design Thinking come metodologia orientata all'innovazione, basata su una prospecctiva esplorativa de liberazione delle idee, e meno focalizzata sull'esecuzione.

Figura 1. Il ruolo del Design Thinking e l'ulteriore procesodi «viluppo dei brevetti».

Fonte: Elaborazione propia
Molte organizzazioni hanno adottato il Design Thinking come metodologia orientata all'innovazione, basata su una prospettiva esplorativa di liberazione delle idee, e meno focalizzata sull'esecuzione; al di là della valutazione delle prestazioni del team, sono necessari più «comportamenti creativi» che si allineino agli obiettivi di innovazione (Chavaglia et al., 2019). Un processo creativo per la generazione di idee tramite il Design Thinking consente quindi e si conforma all'open innovation all'interno dei team organizzativi.

Il processo creativo potrebbe essere incoraggiante - e speranzoso - per l'innovazione. Tuttavia, questo metodo perde lustro nella misura in cui, come parte di una pratica endogena in un'azienda, viene impostato come un processo leggero e senza struttura, che utilizza strumenti che non permettono di analizzare il "progresso creativo» della partecipazione del team (Kempe e Memmert, 2018). In un altro caso, quando si deduce il movimento del diritto e del design nel contesto di una definizione inadeguata dei «diritti di proprietà».

Indubbiamente, le aziende devono introdurre metriche di analisi creativa allineate agli obiettivi per determinare il successo iniziale delle nuove iniziative. L'esercizio di generare idee (correttamente supportato, attraverso un quadro istituzionale robusto e solvibile) aumenta le possibilità di creare innovazione ed essenzialmente assicura la sopravvivenza organizzativa.

I metodi di risoluzione dei problemi (basati sulla generazione di idee) sono iterativi (Buchanan, 1992, p.16), e i loro risultati non sono deterministici. Questo processo non è tradizionalmente misurabile a causa del grado di vulnerabilità informativa, incertezza e potenziale asimmetria, rendendo difficile misurare il valore aggiunto delle azioni. Un corpo di pensiero può essere protetto solo se viene espresso e creato un record (Schönhals, Hepp, e Gipp, 2018, p. 107), creando un processo basato sul Design Thinking che trasforma le informazioni da soggettività creativa a soggettività proiettata.

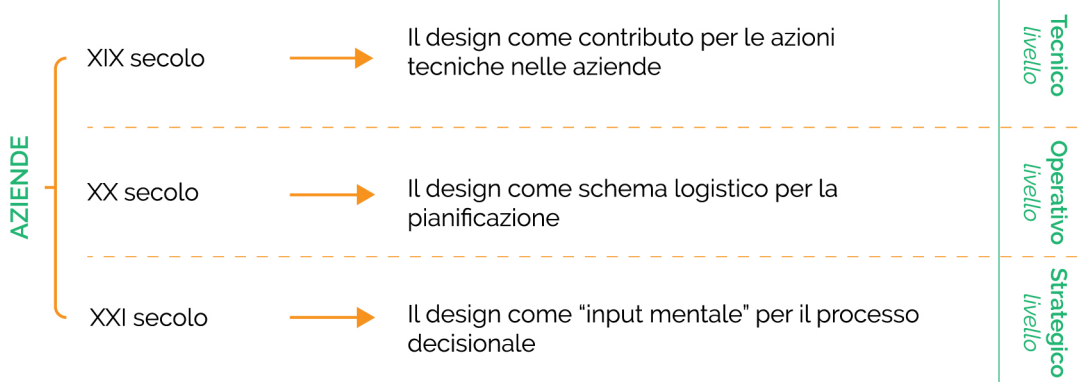

Il bisogno di idee e invenzioni è registrato su (piattaforme fisiche che potrebbero includere riferimenti o timbri di certificazione della proprietà come segno distintivo). Per decenni, questo bisogno crea benefici legati all'innovazione, e con i costi sociali ed economici legati alla concorrenza (Hall, 2007, p. 16). Poi, si pensa che la protezione della proprietà intellettuale per i prodotti motivi e incentivi le aziende a investire in «ricerca e sviluppo» (R\&S) e promuova la diffusione di idee nuove.

\section{Protezione delle idee e dell'innovazione R\&S}

Di conseguenza, l'innovazione e il posizionamento delle istituzioni e delle organizzazioni sono generalmente misurati dal numero di prodotti protetti o di brevetti richiesti e 
divulgati. Settori specifici scelgono spesso di proteggere le loro innovazioni mantenendole segrete. Allo stesso modo, escludono l'intera comunicazione degli elementi essenziali del prodotto, come la definizione del problema e i prototipi generati nel processo (Dwivedi et al., 2021). Tuttavia, come si può aprire il processo quando le prime idee e i primi concetti devono essere tenuti segreti all'interno di un'organizzazione? La loro competitività è messa in pericolo?

Questa strategia di proprietà intellettuale passiva risulta dall'esposizione dell'inventario dell'organizzazione attraverso innovazioni brevettate e pubblicizzate (Schönhals, Hepp, e Gipp, 2018, p.108). Da questa prospettiva, le idee conservate nel tempo arricchiscono la memoria aziendale e il movimento creativo dell'azienda, rendendo queste idee più rilevanti per il mercato industriale.

Attraverso un accordo di licenza di brevetto, la protezione delle idee forma un contratto tra due o più parti che permettono a ciascuna di utilizzare la tecnologia brevettata dell'altra per la propria R\&S (Ylitalo, 2017, p. 94). In questo modo, non solo viene concessa una maggiore libertà di progettazione alle parti, ma c'è anche un aumento cumulativo dell'innovazione (Ylitalo, 2017, p. 85). Un'innovazione è brevettabile se soddisfa i requisiti di novità, utilità e non ovvietà delle soluzioni (Mohr, 2001, p. 5). L'obiettivo è che, insieme alla pubblicazione di un brevetto o altro derivato soggetto a protezione legale e giuridica, ci sia tempo sufficiente per l'organizzazione richiedente per agire e capitalizzare la sua innovazione.

Johnson (2004) sostiene che la divulgazione di oggetti che beneficiano di protezione legale secondo lo schema dei brevetti o altri può essere vista come una risposta alla paura che un'altra persona possa ricevere un brevetto essenziale e dannoso in un'area critica. Dice anche che è iniziato alla fine degli anni '90. Questa pratica è diventata sempre più comune nelle industrie high-tech. La protezione delle invenzioni ha contribuito significativamente all'innovazione, specialmente nei paesi altamente industrializzati, avvantaggiando la produzione fin dalle prime fasi del processo creativo.

\section{Processi creativi e barriere al Design Thinking}

Non è una novità menzionare che le organizzazioni stabiliscono barriere per applicare il Design Thinking, che si concentra sulla misurazione dell'efficienza e della produttività. I comportamenti creativi nell'organizzazione sono difficili da misurare e, in molti casi, non sono osservabili (Sözbilir, 2018). Queste variabili si trasformano in un problema ricorrente poiché il Design Thinking non viene valutato con le metriche tradizionali. Le organizzazioni dovrebbero misurare sia i comportamenti creativi che i comportamenti e le performance (Ylitalo, 2017, p. 65).

Lo sviluppo cognitivo per il problem solving creativo fornisce strumenti di analisi procedurale. Con l'avvento del movimento Gestalt $(1920)^{2}$, che includeva Max Wertheimer, Wolfgang Köhler e Kurt Koffka, sono stati fatti dei contributi al pensiero umano, come i principi olistici di organizzazione dell'informazione. La Scuola di Würzburg propone una comprensione che il problema creativo deve essere risolto

2. La Gestalt fu un movimento che emerse in Germania nel ventesimo secolo. Il termine Gestalt significa "tutto", "forma" o "configurazione", ed è stato ispirato dalla fenomenologia tedesca e dalla scuola di Wurzburg 4 II termine "comportamento umano" è inteso come una posizione che inizia con una reazione che propone e, in molti casi, procede prime dottrine mentali. 
Il Design thinking propone le seguenti fasi: capire, osservare, definire, ideare, creare un prototipo e testare. Tute queste sono sotto la premessa di risolvere problemi centrati sull'utente (cultura dell'innovazione) e di coinvolgere il cliente e tutti i partecipanti al ciclo dell'innovazione (relazioni interattive). usando la direzione del comportamento umano. Questo movimento guida l'influenza sui processi mentali associati al problema-soluzione-comportamento (Wang e Chiew, 2008 , p. 3) come un approccio unico che permette altre correnti interdisciplinari di pensiero nell'inconscio. Questa logica è un meccanismo organizzativo schematico, che permette alle informazioni orientate alla logica del pensiero di essere strutturate per e per raggiungere gli obiettivi (Alfaro et al., 2020).

Infatti, il lavoro mentale si costruisce a partire dalla percezione visiva; cioè, simulazioni visive che proiettano il significato sulle informazioni. L'Università di Stanford ha perseguito un approccio simile, orientato alla creatività dall'esercizio cognitivo e pratico che, a partire dal Design Thinking, crea una grande varietà di risultati e variabili. Le cause principali sono la mancanza di tracciabilità delle idee individuali e l'alta porzione di decisioni soggettive prese nel processo creativo. Pertanto, ogni progresso nel processo di innovazione per essere riconosciuto e valorizzato dall'azienda deve avere la capacità di essere scavato e protetto (Schönhals, Hepp, e Gipp, 2018, p. 110).

Quindi, tenendo conto di queste considerazioni, le informazioni del Design Thinking possono essere soggette alla protezione della proprietà intellettuale nelle prime fasi? Non basta che il gruppo creativo dell'azienda generi idee: bisogna proiettare idee di alta qualità, creando un sistema di informazioni di supporto per i brevetti. Un brevetto può essere considerato una metrica del progresso di R\&S di un'azienda (Schönhals, Hepp, e Gipp, 2018, p. 106). Le organizzazioni hanno scarsi incentivi per il lavoro creativo. Questa categoria è in sospeso nelle aziende il cui management può offrire incorporazioni attraenti riguardo ai benefici della generazione di idee. Le prime fasi creative dello sviluppo del prodotto sono processi giovani per misurare la creatività; usano metriche che ci permettono di determinare efficacemente se un'idea è buona o cattiva (Nelson, 2009, p. 76). Da un lato, questa metrica si basa su processi che misurano il numero di idee e le possibilità di miglioramento e, dall'altro, su risultati che dimostrano il fattore novità (Vargas Hernández, 2010, p. 78).

II Design Thinking richiede che un alto grado di rigore sia incorporato presto nel processo creativo, integrando indicatori e criteri per stimare l'efficacia di un'idea (Meyer e Norman, 2020). Si evidenzia così che il processo creativo richiede più garanzie per dimostrare l'originalità del suo contributo. II Design Thinking propone le seguenti fasi: capire, osservare, definire, ideare, creare un prototipo e testare. Tutte queste sono sotto la premessa di risolvere problemi centrati sull'utente (cultura dell'innovazione) e di coinvolgere il cliente e tutti i partecipanti al ciclo dell'innovazione (relazioni interattive) (Buchanan, 1992, p.11). Perciò, il comportamento e lo sviluppo delle idee richiedono una garanzia di originalità perché queste idee siano protette. $E$, inoltre, contribuisce con qualità specifiche per la registrazione della proprietà intellettuale: a) definire l'origine esatta dell'idea; b) ottenere informazioni accurate che non violino la proprietà dei dati dell'organizzazione; e, c) identificare i diritti dei partecipanti sulle idee create (Teixeira e Ferreira, 2019). Suggerisce di stabilire modelli di misurazione delle idee che si trasformano in metriche di creazione di valore che valutano specificamente i dati quantitativi e qualitativi e valutano la conoscenza ripetitiva del processo.

\section{Pensiero creativo basato su metriche di oggetti legali e di protezione giuridica}

Emma (2005) sostiene che il «valore dell'invenzione» non è il valore di un oggetto di protezione legale e giuridica (brevetto o altro), ma come la qualità di un'idea e il suo valore soggettivo può rappresentare un contributo creativo per il suo inventore. 
e gli utenti. Suggerisce che la metrica per misurare le idee sia proiettata come una metrica di valore basata sui criteri minimi per la registrazione di oggetti di protezione legale e giuridica e la valutazione della capacità organizzativa innovativa. Pertanto, il comportamento e lo sviluppo delle idee richiedono una garanzia di originalità perché queste idee siano protette. $\mathrm{E}$, inoltre, contribuisce con qualità specifiche per la registrazione della proprietà intellettuale: a) definire l'origine esatta dell'idea; b) ottenere informazioni accurate che non violino la proprietà dei dati dell'organizzazione; e, c) identificare i diritti dei partecipanti sulle idee create (Teixeira e Ferreira, 2019). Suggerisce di stabilire modelli di misurazione delle idee che si trasformano in metriche di creazione di valore che valutano specificamente i dati quantitativi e qualitativi e valutano la conoscenza ripetitiva del processo.

Questa iniziativa crea uno strumento di base per misurare l'impatto dei modelli di problem-solving costruendo un record oggettivo e tracciabile dello stato di avanzamento in ogni fase del Design Thinking. Dal momento che l'idea originale può essere verificata, la conseguente protezione legale potrebbe essere basata su innovazioni identificabili sempre considerando alcuni criteri rilevanti per la loro gestione legale e giuridica (Ciani et al., 2016). Se il Design Thinking non può avere successo, viene rapidamente abbandonato per un'altra metodologia di innovazione più facile da seguire. Il comportamento e lo sviluppo delle idee richiedono quindi una garanzia di originalità perché queste idee possano essere brevettate e accedere al registro della proprietà intellettuale: definire l'origine esatta dell'idea, ottenere informazioni accurate che non violino la proprietà dei dati dell'organizzazione e identificare i diritti dei partecipanti sulle idee create (Teixeira e Ferreira, 2019). Suggerisce di stabilire modelli di misurazione delle idee che si trasformano in metriche di creazione di valore che valutano specificamente i dati quantitativi e qualitativi e valutano la conoscenza ripetitiva del processo.

\section{Pensiero creativo basato sulle metriche dei brevetti}

Poege et al. (2019) sostengono che il «valore dell'invenzione» non è il valore di un brevetto, ma la qualità di un'idea e il suo valore soggettivo possono rappresentare un contributo creativo per il suo inventore e gli utenti. Suggerisce che la metrica per

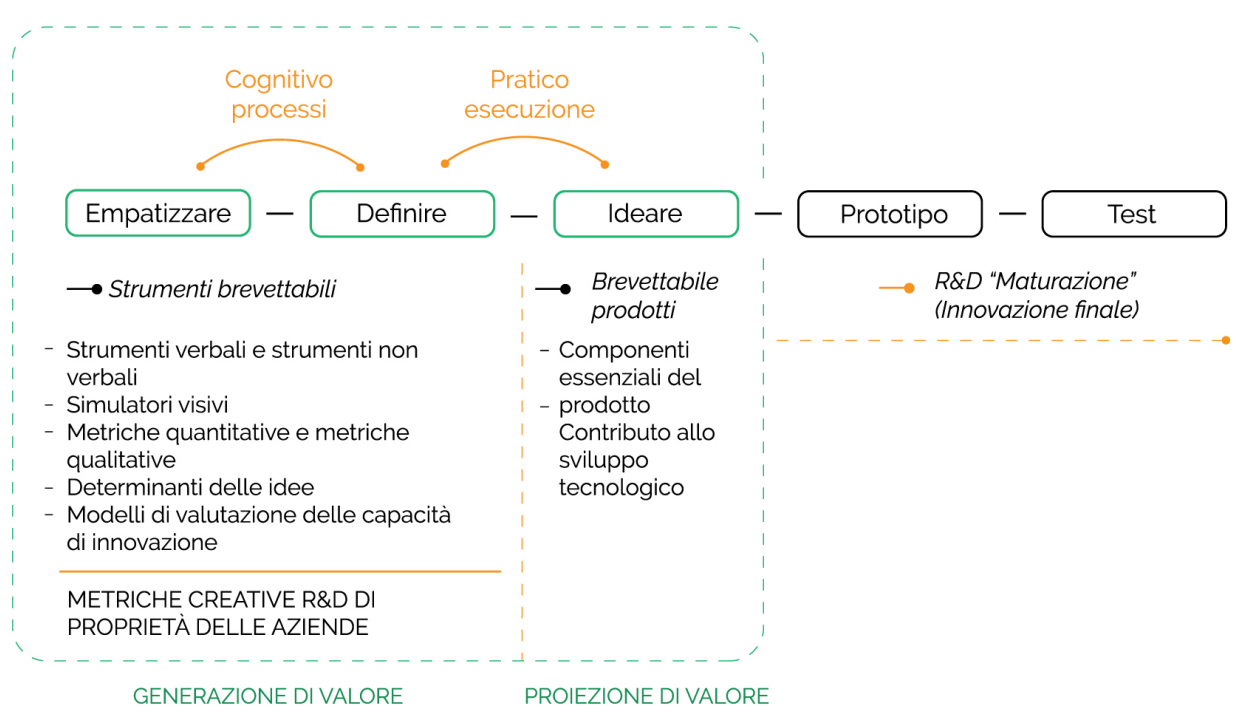


misurare le idee sia proiettata come una metrica di valore basata sui criteri minimi per la registrazione dei brevetti e la valutazione della capacità organizzativa innovativa.

D'altra parte, Ylitalo (2017, p. 92) assume che l'importanza del sistema dei brevetti e la conseguente analisi dei dati è stata enfatizzata, per la maggior parte, nella gestione dell'alta tecnologia. I processi diventano sempre più complessi e il ciclo dell'innovazione si accorcia (Yoon e Park, 2004, p. 38). I processi brevettuali legati alla tecnologia spingono alla digitalizzazione delle fasi del Design Thinking, assicurando il contenuto delle informazioni che determinano i partecipanti come contributori individuali degli utenti nei processi collaborativi (autore e partecipanti) e il livello di contributo e novità.

Questa iniziativa crea uno strumento di base per misurare l'impatto dei modelli di problem-solving costruendo un record oggettivo e tracciabile dello stato di avanzamento in ogni fase del Design Thinking. Poiché l'idea originale può essere verificata, i brevetti risultanti possono quindi essere basati su innovazioni riconoscibili (Ciani et al., 2016). Se il Design Thinking non può dimostrare il successo, viene rapidamente abbandonato per un'altra metodologia di innovazione più facile da tracciare.

L'uso di metriche nel processo di Design Thinking, basato sulla tecnologia digitale, ci incoraggia a considerare come gli strumenti di misurazione possono essere modificati e personalizzati per proteggere e garantire l'integrità dei dati. Questi strumenti hanno vari approcci categoriali: non verbale, rilevato da telecamere RGB regionali, telecamere IR o sensori a ultrasuoni (Schönhals, Hepp e Gipp, 2018, p. 107), che catturano i metodi della comunicazione umana, come gesti, espressioni facciali e movimenti del corpo. Questi completano le informazioni semantiche dell'espressione verbale. Dall'altro lato, viene modellato o costruito, concentrandosi sulla gestione di scanner tridimensionali e modelli CAD. A seconda della definizione del problema e della soluzione per la soluzione, questi approcci sono classificati come le due sfide più significative nella registrazione dei risultati durante un processo di Design Thinking (Schönhals, Hepp, e Gipp, 2018, p. 107).

Di conseguenza, gli strumenti digitali sono uno strumento di protezione della proprietà intellettuale che espande le possibilità di brevettazione in modo rigoroso, sistematico e in tempo reale nelle fasi creative dei processi. Un sistema di misurazione e valutazione delle idee nelle fasi creative può portare a un sistema brevettabile e a un know-how che rende un'azienda altamente competitiva.

Dato che l'idea originale può essere verificata, la protezione legale risultante potrebbe essere basata su innovazioni identificabili sempre considerando alcuni criteri rilevanti per la sua gestione legale e giuridica (Ciani et al., 2016). Se il Design Thinking non può dimostrare il successo, viene rapidamente abbandonato per un'altra metodologia di innovazione più facile da tracciare.

\section{Conclusioni}

Il nuovo ruolo del design e della tecnologia nella cultura contemporanea non si trova nella comprensione corrente della filosofia della tecnologia, ma nel significato della conoscenza e nel modo di usare le capacità creative del pensiero umano nell'organizzazione. L'idea centrale nella creazione dell'innovazione brevettabile è che l'esercizio creativo del Design Thinking sia proiettato sugli obiettivi organizzativi, rivelando così 
categorie ampiamente misurabili e percepibili riguardo ai benefici competitivi ed evolutivi dell'azienda.

Il Design Thinking richiede che un maggior rigore sia sistematizzato e incorporato nel processo creativo attraverso metriche che generano dati quantitativi sull'ordine delle variabili e degli indicatori. In altre parole, il lavoro dettagliato per le informazioni ottenute dal processo creativo deve essere rafforzato. L'individualità e la libertà di pensiero del team di lavoro devono essere comprese perché la metrica non si comporterà necessariamente come l'intero team creativo. In breve, sia i processi di valutazione che gli incentivi devono essere criteri di applicazione collettiva, dove i valori collettivi sono valutati come i risultati dell'intera azienda.

II sistema creato riguardo agli oggetti soggetti a protezione legale come i brevetti o altro, fornisce incentivi e motivazioni per investire in R\&S e promuove la diffusione di idee nuove, dando un risultato vantaggioso per le organizzazioni. II Design Thinking deve rafforzare la sua iniziativa originale verso la generazione di informazioni misurabili che contribuiscono all'innovazione e all'ottenimento di prodotti innovativi. Infine, I'alta direzione di un'organizzazione deve concentrare le sue azioni sull'essere umano e dirigere le sue strategie di business tenendo presente il comportamento e la motivazione del suo team di lavoro.

\section{Riferimenti}

Alfaro, M., Vargas, M., Gutierrez, S., Ternero, R. e Sabattin, J. (2020). Conceptual Framework for the Strategic Management: A Literature Review-Descriptive. Journal of Engineering, 2020.

Buchanan, R. (1992). Wicked problems in design thinking. Design issues, 8 (2).

Chavaglia, J., Filipe, J. e Bento Caleiro, A. (2019). Creativity and innovation: A contribution of behavioral economics. International Journal of Innovation Studies, 3 (1), 12-21.

Ciani, O., Armeni, P., Boscolo, P., Cavazza, M., Jommi, C. e Tarricon, R. (2016). De innovatione: The concept of innovation for medical technologies and its implications for healthcare policy-making. Health Policy and Technology, 5 (1), 47-64.

Dorst, K. (2019). Design beyond Design. She Ji: The Journal of Design, Economics, and Innovation, 5 (2), 117-127.

Dwivedi, Y. et al. (2021). Setting the future of digital and social media marketing research: Perspectives and research propositions. International Journal of Information Management, 59, 102-168.

Gassmann, O. e Bader, M. (2006). Patentmanagement. Wiesbaden: Springer Gabler.

Hall, BH. (2007) Patents and patent policy. Oxford Review of Economic Policy, 23 (4), 568-587).

Johnson, JP. (2004) Defensive publishing by a leading firm. Working paper. Ithaca: Cornell University Press.

Kempe, M. e Kempe, D. (2018). "Good, better, creative»: the influence of creativity on goal scoring in elite soccer. Journal of Sports Sciences, 36 (3), 1-5.

Meyer, M. e Norman, D. (2020). Changing Design Education for the 21st Century. She Ji: The Journal of Design, Economics, and Innovation, 6 (1), 13-49. 
Mohr, J. (2001). Marketing of high-technology products and innovations. Upper Saddle River: Prentice-Hall.

Nelson B. A., Yen J., Wilson J. O. e Rosen D. (2009). Refined Metrics for measuring ideation effectiveness. Design Studies, 30 (6), 737-743.

Poege, F., Harhoff, D., Gaessler, F. e Baruffaldi, S. (2019). Science quality and the value of inventions. Sciences Advances, 5 (12). doi: 10.1126/sciadv.aay7323

Royalty A. e Roth B. (2015). Developing Design Thinking Metrics as a Driver of Creative Innovation. New York: Springer International Publishing.

Schmiedgen, J., Spille, L-, Köppen, E., Rhinow, H. e Meinel, C. (2016). Measuring the impact of design thinking. In Plattner $\mathrm{H}$, Meinel C, e Leifer L (eds.), Design Thinking Research: Making Design Thinking Foundational, 157-170. Switzerland: Springer International Publishing.

Schönhals, A., Hepp, T. e Gipp, B. (2018). Design Thinking using the Blockchain: Enable Traceability of Intellectual Property in Problem-Solving Processes for Open Innovation. CryBlock'18: Proceedings of the 1st Workshop on Cryptocurrencies and Blockchains for Distributed Systems, 105-110.

Sözbilir, F. (2018). The interaction between social capital, creativity and efficiency in organizations. Thinking Skills and Creativity, 27, 92-100.

Teixeira, A. e Ferreira, C. (2019). Intellectual property rights and the competitiveness of academic spin-offs. Journal of Innovation er Knowledge, 4 (3), 154-161.

Totten, J. e Zhai, M. (2014). Recent developments in the interplay between patents and standards-setting. Chicago: IEEE.

Turnali, K. (2015/05/10). What Is Design Thinking? Forbes. Recuperato da: https:// www.forbes.com/sites/sap/2015/05/10/what-is-design-thinking/?sh=6f12bcd8471f

Ulrich, K. (2012). Diseño y desarrollo de productos. México: The McGraw-Hill Companies.

Vargas-Hernandez N., Shah J). e Smith SM. (2010). Understanding design ideation mechanisms through multilevel aligned empirical studies. Design Studies, 31, 382- 410.

Wang, Y., e Chiew, V. (2008). On the cognitive process of human problem-solving. Cognitive Systems Research, 11 (1), 81-92.

Ylitalo, P. (2017). Value Creation Metrics in Systematic Idea Generation. Acta Univiversitatis Ouluensis, C 611.

Yoon, B. e Park, Y. (2004). A text-mining-based patent network: analytical tool for high technology trend. The Journal of High Technology Management Research, 15 (1), 37-50. 